\title{
From theory to practice in general practice audit
}

\author{
Mike Pringle
}

Hospital colleagues can reflect on a long history of case based auditing. Through postmortem examinations (perhaps the ultimate audit), grand rounds, and perinatal morbidity meetings, secondary care doctors have developed the ground rules of openness and mutual support in critical case analysis, and they continue with this work in the confidential enquiry into perioperative deaths (CEPOD). ${ }^{1}$ They have no real tradition of cohort based audits, although they have of course extensively researched on groups of patients.

Primary care, on the other hand, has had no such track record of auditing. Though its research had been a hesitant flowering for several decades, until the 1980 s primary care auditors were rare, delicate blooms. The first sightings were in relation to vocational training, with trainers' practices expected to audit their medical records to show a sufficient standard of record keeping. This was clearly linked with peer group status and the financial rewards of being a vocational trainer and was therefore accepted as a necessary inconvenience. Examples of audits improving quality of care are hard to come by, but it is widely accepted anecdotally that the record keeping requirements for accreditation for training had a dramatic and lasting effect on both the standards of the records and the manner in which they were viewed and used within the practices.

In 1983 the innovators of vocational training, embodied in the then chairman of the council of the Royal College of General Practitioners, Donald Irvine, developed the college's quality initiative. ${ }^{2}$ In primary care as a whole it was largely greeted with pious hopes and atavistic apathy. However, this is the natural response to radical thinking, and the members of the council began to audit their practices and publish the results. ${ }^{34}$

The extraordinary foresight of the quality initiative is demonstrated by its two principal aims. "Within general practice as a whole each of us should be able to achieve the following broad aims within the next ten years:

(1) To be able to say, at any moment in time, what the content of our work is and therefore what services each of us provides

(2) To incorporate standard setting and performance review as an integral and effective part of our professional lives."

Department of General Practice, Nottingham University Medical School, Nottingham Mike Pringle, senior lecturer

\section{Responding to the quality initiative}

Firstly, it seems most appropriate to examine the performance of the college. It set itself up as the flagship for quality, so how has it fared? By 1985 the college had produced a working party report What Sort of Doctor? $?^{5}$ It set out a scheme for exchange practice visits in which both those visited and the visitor could look at the practice's performance and learn from it. As a first practical guide to peer review it was a radical concept and it floundered for two reasons: its culture preceded its time, and it required substantial effort for little immediate, secular reward.

The What Sort of Doctor? deliberations were not, however, wasted. The college built on them to develop the principles and, finally, in 1989 , to achieve the reality of fellowship by assessement. ${ }^{6}$ Many college members were and are dissatisfied with the traditional route to fellowship. This dissatisfaction stems from an appearance of arbitrary allocation: "It's who you know, not what you do," and its emphasis on service to the profession rather than to patients. A series of essential criteria were developed, all based on patient care, which are both objective and auditable. An applicant knows in advance the standard to be met, and all 65 assessment criteria must be met.

Although this takes the concept of audit into target standards and accreditation, fellowship by assessment stays true to the principles of auditing. It is a voluntary, peer group activity which is educational and intended to stimulate and show quality of care in general practice. Recently there has been an increasing clamour for membership by assessment, and proposals for an alternative to the MRCGP examination will be put forward by the college this autumn.

\begin{tabular}{c}
\hline ". . . there has been an \\
increasing clamour for \\
membership by assessment ..."
\end{tabular}

Throughout the 1980 s the concurrent tightening up of vocational training practice visits and assessments has helped to create a culture of critical evaluation in many practices in Britain. This has been led by the Royal College of General Practitioners and the General Medical Services Committee of the BMA through the Joint Committee for Postgraduate Training for General Practice. 
The GMSC recently made known the results of its comprehensive survey of general practitioners' attitudes ${ }^{7}$; only $33 \%$ of about 25000 respondents disagreed with the statement that "an accreditation system in general practice is long overdue."

It seems clear that the opinion formers in primary care, including the college, have been substantially successful in the task of creating both the climate and methods for audit which have facilitated the auditing revolution. For if auditing is to have an end point it must be to raise quality and show that quality for all patients. This must eventually include, in my view, auditing against standards which are both targets, as in fellowship by assessment, or minimum standards, as in re-accreditation.

If the profession has been the first parent of auditing, the second, more controversial, progenitor was the NHS reforms, ${ }^{8}$ whose only provision to achieve an almost universal welcome was that concerned with medical audit. ${ }^{9}$ A cynic might observe that the welcome for this working paper was more a reflection of ignorance than insight or perhaps a case of the other proposals distracting attention while medical audit was infiltrated. But if audit slipped in like a wolf in sheep's clothing, this might be not necessarily be deleterious.

The NHS reforms crystallised another boost for audit - the espousal of consumerism. Though patient power is still muted in the health service, the quality provisions in secondary care contracts, the patient's charter, and the emphasis on patient choice have all created a climate in which it is insufficient to believe that we deliver a quality service. We are now in a position where we must show that we deliver a quality service.

Medical audit . . . is set to

become the norm throughout primary care.

All our experience so far, therefore, suggests that auditing went through its innovatory phase powered by the professional conscience of the leaders and a substantial carrot in the form of fellowship by assessment. Then the concept was imposed, but without the substantial opposition that met the other imposed changes of the NHS reforms. Like computerisation, medical audit has entered the everyday culture of sufficient practices to be respectable and now is set to become the norm throughout primary care. In order to see whether such an optimistic view is tenable I will look at my own experience.

Practice audit: a personal view

As a partner in what might be referred to as an auditing practice I have had experience of auditing prescribing over eleven years as part of formulary development and maintenance. We never called it "auditing" but we agreed a prescribing policy (a formulary), recorded and reviewed our performance (regular prescribing meetings), and altered our behaviour. We never forbade any doctor from prescribing outside the formulary, but we expected such prescriptions to be justifiable in the practice meeting.

As a dispensing practice we had a strong financial motive for auditing our prescribing. When, eight years ago, we entered our next phase of medical student and vocational trainee audits it was because audit was seen as a part of our educational responsibility. The partnership started clinical auditing with a vengeance five years ago, and our motivation was greatly enhanced by applying for fellowship by assessment. We have twenty protocols which are "published" in a booklet on every doctor's desk, and we "record" the results of our audits in our practice report. ${ }^{10} \mathrm{I}$ mention our motivation here because of its importance. All auditing exacts a price, and the reward, which is not necessarily financial and is more likely to entail enhanced self esteem, must be sufficient to outweigh the costs in time, energy, and resources. A practice only has so much libido, and it needs to apply it where it gets the best return.

We are now finishing our first year of organised auditing of significant events. "This is a new departure in two ways. Firstly, it is our first attempt at structured case based auditing. We note down a number of predetermined events - every myocardial infarction, stroke, parasuicide, urgent visit for asthma or diabetes, patient complaint, etc and any other events we think are important. At a bimonthly meeting we select patients and discuss in detail the care they received. We might look at the interval from presentation to diagnosis of a newly diagnosed cancer or an administrative mix up which led to a patient becoming angry. Auditing significant events leaves behind such notions as groups, samples, means, and norms. It is highly unscientific, being based on a few selected cases. But it is powerful in achieving the very results which conventional auditing often neglects - it deals with the immeasurable, the qualitative aspects of care, and by provoking powerful emotional responses it is forcefully linked with changes in behaviour.

\begin{tabular}{c}
\hline motivation is most clearly \\
related to quality.
\end{tabular}

Secondly, motivation is most clearly related to quality. We positively enjoy, if sometimes in a masochistic way, the interchange of clinical beliefs and behaviour, and we value the mutual support and friendship that it generates. This in turn helps us to have more confidence in confronting our failings and seeking help from our partners.

This personal odyssey is not irrelevant to the subject of this article. It illustrates that 
auditing has been happening, albeit until recently, in limited numbers of practices. It also shows that the methods are already quite advanced and are being developed all the time. And, lastly, it shows that nothing will be achieved unless there is motivation - and that motivation is personal, variable, and elusive.

\section{Practice audit: past and future}

Over the past decade opinion formers in the guise of vocational trainers, the Royal College of General Practitioners, and innovative practices have been creating a culture and methodology for audit. This was capitalised on by the NHS reforms, which have added two vital ingredients - organisation and money - at what I believe we shall come to see as a fortuitous time. What has been happening in recent months?

For the past two years I have been involved with Lincolnshire Medical Audit Advisory Group, ${ }^{12}$ firstly, helping in its birth and then, for the past year, helping it to thrive in its infancy. We adopted a structure in which a cadre of audit visitors, called audit ambassadors, are employed on a sessional basis to visit and help practices. Each ambassador has just over ten practices and the number of visits a year to each practice varies. Practices which are literate in audit might need one visit a year; others might need a series of visits. The ambassadors are supported by an audit administrator who, as a former practice manager, visits many practices to help in the practicalities of auditing.

At the end of the first year only one practice of the 108 in Lincolnshire has not been visited. Of the others, $57(51 \%)$ have done at least one audit to the point of implementing action. Many of these will have a wide portfolio of audits, including repeat audits which complete the audit cycle. Some practices have not reaudited yet, but in the first year that expectation may be too high. Another $32(30 \%)$ practices are undertaking their first audit at present, and a further $17(16 \%)$ say they are at the planning stage. That leaves three in whom no auditing is happening.

From the ambassadors' discussions with practices 242 completed audits have been recorded, with a further 138 in progress and 191 planned. These range from an audit of acupuncture to one on $x$ ray examinations, covering nearly 150 different topics. This is a most gratifying response in the first year, but doubts remain. Can the momentum be maintained? Will the effort being expended result in real improvements in quality? Will the government continue to support the peer led, educational nature of the initiative, and will it begin to reduce the resources?

\section{Conclusion}

There has been an exponential growth in medical auditing in the past two years, but this has been from a very low activity base. Though the state of auditing now is much higher than anybody had good reason to predict only two years ago, it is still not sufficient to deem the battle won. Auditing is still an "add on" to practice, not a regular part of the everyday care of our patients.

In the near future it is to be hoped that the real potential of general practice computers to help in medical audit will be realised. ${ }^{13}$ They can of course save only time in data collection and presentation. Nothing can make the process of changing behaviour painless, but if the drudgery can be relieved auditing time can be more usefully employed in looking at, talking about, and exploring the real kernel that is, quality care for every patient in every practice.

1 Buck N, Devlin HB, Lunn JN. The report of a confidential enquiry into perioperative deaths. London: Nuffield Provincial Hospitals Trust and King Edward's Hospital Fund for London, 1987

2 Royal College of General Practitioners. Quality in general practice: policy statement 2. London: RCGP, 1985.

Quality of care initiative by members of council. London: RCGP, 1984.

4 Quality of care initiative by members of council. London RCGP, 1986.

5 Working Party. What son of doctor? London: RCGP, 1985 (Report from general practice 23.)

6 Royal College of General Practitioners. Fellowship by assessment. London: RCGP, 1990. (Occasional paper 50 .)

7 Electoral Reform Ballot Services. Your choices for the future. $A$ survey of GP opinion. UK Report. London: Electoral Reform Ballot Services, 1992

8 Secretaries of State for Health, Wales, Northern Ireland, and Scotland. Working for patients. London: HMSO, 1989. (Cmnd 555.)

9 Secretaries of State for Health, Wales, Northern Ireland and Scotland. Medical audit. Working paper 6. London: HMSO, 1989.

10 Pringle M. Practice reports. In: Mariker M, ed. Medical audit and general practice. London: BMJ, 1990:196-223. Pringle M. Auditing of significant events. Practice Manager 1991;May:5.

12 Department of Health. Medical audit in the family health services. London: HMSO, 1990. (FP (90) 8.)

13 Royal College of General Practitioners Computer Working Party. General practice computers: the clinical issues. London: RCGP, 1992. 